\title{
WHICH FUNCTIONS PRESERVE CAUCHY LAWS?
}

\author{
GERARD LETAC ${ }^{1}$
}

\begin{abstract}
A real random variable $X$ has a Cauchy law $C(a, b)$ if its density is $b \pi^{-1}\left[(x-a)^{2}+b^{2}\right]^{-1}$, with $b>0$ and $a$ real. Let $f$ be a measurable function such that $f(X)$ also has a Cauchy law for any $a$ and $b$. We prove that there exist $\alpha$ real, $k>0, \varepsilon= \pm 1$ and a singular positive bounded measure $\mu$ on $\mathbf{R}$ such that for almost all $x$ of $\mathbf{R}$
\end{abstract}

$$
\varepsilon f(x)=k x+\alpha-\lim _{y \downarrow 0} \int_{-\infty}^{+\infty} \frac{(x+i y) \gamma+1}{x+i y-\gamma} \mu(\alpha \gamma) .
$$

Conversely, every function $f$ with the preceding form is such that $f(X)$ has a Cauchy law when $X$ has a Cauchy law.

Furthermore, we prove that such a function preserves Lebesgue measure when $k=1$, generalising a well-known Pólya and Szegö result.

1. Introduction. Recently, attention has been called to some transformations of the real line $\mathbf{R}$ preserving either Lebesgue measure, or a Cauchy law, or the whole set of the Cauchy laws.

R. L. Adler and B. Weiss [1] have studied the ergodic properties of the so-called "Boole function" $f(x)=x-x^{-1}$ which preserves Lebesgue measure of $\mathbf{R}$. Such an $f$ is not the only rational function preserving Lebesgue measure, and we find in Pólya and Szegö [7] a complete characterisation of such functions $f$ : there exist $\alpha$ real, $\varepsilon= \pm 1$, an integer $n \geqslant 0$, numbers $\gamma_{1}<\cdots<\gamma_{n}$ and positive numbers $\mu_{1}, \mu_{2}, \ldots, \mu_{n}$ such that

$$
\varepsilon f(x)=x+\alpha-\sum_{i=1}^{n} \frac{\mu_{i}}{x-\gamma_{i}} .
$$

A real random variable $X$ has a Cauchy law $C(a, b)$ if its density is $b \pi^{-1}\left[(x-a)^{2}+b^{2}\right]^{-1}$, with $b>0$ and $a$ real. Boole function offers another interesting property: if the random variable $X$ has a Cauchy law $C(0,1)$, then $\frac{1}{2}\left(X-X^{-1}\right)$ has also a Cauchy law $C(0,1)$ : a nice geometrical proof can be obtained using the classical interpretation of $C(0,1)$ as the stereographic projection on the line of the uniform distribution on a circle. This fact can be extended to the functions defined by (1): if $X$ has a Cauchy law, this is also true for $f(X)$, with possibly different parameters. Other rational functions share with the functions defined by (1) the property of preserving the whole

Received by the editors December 10, 1976 and, in revised form, March 16, 1977.

AMS (MOS) subject classifications (1970). Primary 28A65, 30A76, 60E05; Secondary 60J65, $60 \mathrm{~J} 10$.

Key words and phrases. Measure preserving transformations, Poisson kernel, Cauchy laws.

'The main part of this research has been done while the author was a member of the "Ecole d'Été de Probabilité 1976" at Saint-Flour, France. 
set of Cauchy laws, in particular real projectivities $h(x)=(a x+b) /(c x+$ $d$ ), where $a, b, c, d$ are real and $a d-b c \neq 0$; this fact plays an important role in characterisations of Cauchy laws (see E. J. Williams [10], F. Knight [5] and F. Knight and P. A. Meyer [6]). Collecting these results, we see that if:

$$
\varepsilon f(x)=k x+\alpha-\sum_{c=1}^{n} \frac{\mu_{i}}{x-\gamma_{i}}
$$

where $k$ and $\mu_{i}$ are $>0, \alpha$ and $\gamma_{i}$ are real and $\varepsilon= \pm 1$, then $f$ preserves Cauchy laws.

It is possible to give an elementary, but lengthy, proof of the fact that if $f$ is rational and preserves the whole set of Cauchy laws, it has the form described by (1). But that will be also a trivial consequence of the theorem of our paper.

The next observation is a work by J. H. B. Kempermann [4] considering transformations of the real line which are restrictions to $\mathbf{R}$ of meromorphic functions $f(z)$ such that

$$
\varepsilon f(z)=k z+\alpha-\sum_{s} p_{s}\left[\frac{1}{z-\gamma_{s}}+\frac{1}{\gamma_{s}}\right]
$$

where $\varepsilon= \pm 1,0<k<1, p_{s}>0, \alpha$ and $\gamma_{s}$ are real, $\left\{\gamma_{s}\right\}$ has no finite accumulation point and $\sum p_{s} \gamma_{s}^{-2}<\infty$. J. H. B. Kempermann announces that $f$ preserves one Cauchy law if $f$ has no real fixed point $z_{0}$ such that $\left|f^{\prime}\left(z_{0}\right)\right|<1$.

So we are led to the following question: what are the real measurable functions $f$ of $\mathbf{R}$ such that the random variable $f(X)$ has a Cauchy law when $X$ has a Cauchy law? First, it is necessary to state the following result, in order to give a neat answer:

Proposition. Let $\mu$ be a bounded singular positive measure $\mu$ on $\mathbf{R}$. Then:

$$
\lim _{y \downarrow 0} \int_{-\infty}^{+\infty} \frac{(x+i y) \gamma+1}{x+i y-\gamma} \mu(d \gamma)
$$

exists and is real for almost all real $x$.

THEOREM. Let $f$ be a measurable function from $\mathbf{R}$ to $\mathbf{R}$ then the following properties are equivalent:

(i) For any random variable $X$ with a Cauchy law, the random variable $f(X)$ has a Cauchy law.

(ii) There exist $\varepsilon= \pm 1, \alpha$ real, $k$ nonnegative and a bounded singular positive measure $\mu$ on $\mathbf{R}$ such that for almost all $x$ of $\mathbf{R}$

$$
\varepsilon f(x)=k x+\alpha-\lim _{y \downarrow 0} \int_{-\infty}^{+\infty} \frac{(x+i y) \gamma+1}{(x+i y)-\gamma} \mu(d \gamma) .
$$

Furthermore, if $\tilde{f}$ is defined in the upper half complex plane by

$$
\varepsilon \tilde{f}(\omega)=k \omega+\alpha-\int_{-\infty}^{+\infty} \frac{\omega \gamma+1}{\omega-\gamma} \mu(d \gamma),
$$


then the law of $f(X)$ is $C(A, B)$, with $A=\operatorname{Re} \tilde{f}(a+i b)$ and $B=\mid \operatorname{Im} \tilde{f}(a+$ $i b) \mid$, when the law of $X$ is $C(a, b)$.

Let us insist on the fact that we characterise in this theorem the functions preserving the whole set of Cauchy laws. That would be an entirely different problem to describe the set of functions changing a fixed Cauchy law in another Cauchy law, or the set of functions preserving one given Cauchy law. An immediate consequence of the theorem is that for $f$ and $\tilde{f}$ as in the preceding statement with $\varepsilon=+1, f$ preserves $C(a, b)$ if and only if $a+i b$ is a fixed point of $f$. Sufficient conditions for the existence of such a fixed point are given in [4].

As the Theorem shows, the knowledge of $f$ gives the knowledge of $\tilde{f}$. Now it is not difficult to check that $\varepsilon, k, \alpha$ and $\mu$ are completely determined by $\tilde{f}$; any standard text can be consulted: [2], [8] or [9].

The Theorem enables us to reach a bigger class of functions than the Pólya-and-Szegö ones described by (2), which preserve Lebesgue measure on R.

COROLlaRY. Let $\varepsilon= \pm 1, \alpha$ real and a bounded singular positive measure $\mu$ on $R$. Then $f(x)$, defined by

$$
\varepsilon f(x)=x+\alpha-\lim _{y \downarrow 0} \int_{-\infty}^{+\infty} \frac{(x+i y) \gamma+1}{x+i y-\gamma} \mu(d \gamma)
$$

preserves Lebesgue measure on $\mathbf{R}$.

We shall prove this corollary in $\$ 6$. As an example, we have that

$$
f(x)=x+\operatorname{tg} x
$$

preserves Lebesgue measure, since $\operatorname{tg} \omega$ is equal to

$$
\sum_{n=-\infty}^{+\infty} \frac{\omega \pi\left(n+\frac{1}{2}\right)+1}{\pi\left(n+\frac{1}{2}\right)-\omega} \cdot \frac{1}{\pi^{2}\left(n+\frac{1}{2}\right)^{2}+1},
$$

a classical fact, and

$$
\mu=\sum_{n=-\infty}^{+\infty} \frac{1}{\pi^{2}\left(n+\frac{1}{2}\right)^{2}+1} \delta_{\pi\left(n+\frac{1}{2}\right)}
$$

is a bounded singular positive measure on $\mathbf{R}$, with $\delta_{a}$ unit mass in $a$. Of course, we do not mean in the corollary that every function $f$ preserving Lebesgue measure has the indicated form. Taking $f(x)=x$ if $|x|>1$ and $f(x)=-x$ if $|x| \leqslant 1$, one can prove that such an $f$ does not belong to the class described in the corollary.

2. Notations and preliminaries. We denote by $P$ the open upper half complex plane, that is $\{\omega ; \operatorname{Im} \omega>0\}$ and by $U$ the open unit disk, that is $\{z$; $|z|<1\}$. The circle $\mathbf{T}$ is $\mathbf{R} / 2 \pi \mathbf{Z}$. If $\omega$ belongs to $P$ the measure $\mu_{\omega}(d x)$ on $\mathbf{R}$ is 


$$
\mu_{\omega}(d x)=\frac{1}{\pi} \cdot \frac{\operatorname{Im} \omega}{(x-\operatorname{Re} \omega)^{2}+(\operatorname{Im} \omega)^{2}} d x,
$$

that is to say a Cauchy law $C(\operatorname{Re} \omega, \operatorname{Im} \omega)$. As is well known, the Fourier transform of $\mu_{\omega}$ is given by:

$$
\int_{-\infty}^{+\infty} \exp (i t x) \mu_{\omega}(d x)=\exp (i t \omega)
$$

for all positive $t$.

If $\tilde{f}: P \rightarrow C$, we define $G \tilde{f}: U \rightarrow \mathbf{C}$ by

$$
G \tilde{f}(z)=\tilde{f}\left(i \frac{1+z}{1-z}\right) .
$$

Conversely if $\tilde{g}: U \rightarrow \mathrm{C}$, we define $F \tilde{g}: P \rightarrow \mathrm{C}$ by

$$
F \tilde{g}(\omega)=g\left(\frac{i \omega+1}{i \omega-1}\right) .
$$

If $z$ belongs to $U$ and $\theta$ to T, the Poisson kernel $P_{z}(\theta)$ is $\operatorname{Re}\left[\left(e^{i \theta}+z\right) /\left(e^{i \theta}-\right.\right.$ z)].

Proof of THE Proposition. If the measure $\mu$ is singular positive and bounded on $\mathbf{R}$, the function $\phi$ on $P$ :

$$
\phi(\omega)=\int_{-\infty}^{+\infty} \frac{\omega \gamma+1}{\omega-\gamma} \mu(d \gamma)
$$

gives the function $G \phi$ on $U$ :

$$
G \phi(z)=i \int_{-\pi}^{+\pi} \frac{e^{i \theta}+z}{e^{i \theta}-z} \nu(d \theta)
$$

where $\nu$ is the singular positive measure on $\mathbf{T}$ carried from $\mu$ by

$$
e^{i \theta}=\frac{i \gamma+1}{i \gamma-1} .
$$

Since $\operatorname{Im} G \phi(z)=-\int_{-\pi}^{+\pi} P_{z}(\theta) \nu(d \theta)$ the Theorem 11.10 of [8] implies that

$$
\operatorname{Im} G \phi\left(r e^{i \theta}\right) \underset{r \uparrow 1}{\rightarrow} 0 \text { for almost all } \theta \text { of } \mathbf{T},
$$

since $\nu$ is singular.

We show now that $\lim _{r \uparrow 1} G \phi\left(r e^{i \theta}\right)$ exists for almost all $\theta$. Since

$$
\operatorname{Im} G \phi(r)<0
$$

in $U$, Theorem 3.2 of [2] implies that $G \phi(z)$ belongs to the Hardy space $H^{p}$ $(0<p<1)$ of analytic functions in $U$, that is to say:

$$
\lim _{r \uparrow 1} \int_{-\pi}^{+\pi}\left|G \phi\left(r e^{i \theta}\right)\right|^{p} d \theta<\infty .
$$

The Theorem 2.2 of [2] gives the existence of $\lim _{r \uparrow 1} G \phi\left(r e^{i \theta}\right)$ for almost all $\theta$ of T. So $\lim _{y \downarrow 0} \phi(x+i y)$ exists and is real for almost all real $x$.

3. A Lemma. The proof of the Theorem on a simple lemma which allows one to jump from harmonicity to analyticity: 
LemMa. Let $\Omega$ be a connected open subset of the complex plane and $h$ a complex function defined on $\Omega$ such that $\exp [\operatorname{th}(z)]$ is harmonic in $\Omega$ for all $t>0$. Then $h(z)$ is either analytic or antianalytic in $\Omega$.

Proof of THE Lemma. Clearly, exp(th) harmonic for all positive $t$ implies that $h$ is continuous in $\Omega$. If $z_{0}$ in $\Omega$ and $r>0$ are such that $\left\{z ;\left|z-z_{0}\right|<r\right\}$ $\subset \Omega$, we can write:

$$
\begin{aligned}
0 & =\frac{1}{2 \pi} \int_{-\pi}^{+\pi} \frac{1}{t}\left[\exp t\left(h\left(z_{0}+r e^{i \theta}\right)-h\left(z_{0}\right)\right)-1\right] d \theta \\
& \rightarrow \frac{1}{2 \pi} \int_{-\pi}^{+\pi}\left[h\left(z_{0}+r e^{i \theta}\right)-h\left(z_{0}\right)\right] d \theta,
\end{aligned}
$$

since we can exchange integral and $\lim _{t \downarrow 0}$ by dominated convergence, $h$ being continuous. Hence $h$ itself is harmonic in $\Omega$. From the identity:

$$
\exp (-t h) \frac{\partial^{2}}{\partial z \partial \bar{z}} \exp (t h)=t \frac{\partial^{2}}{\partial z \partial \bar{z}} h+t^{2} \frac{\partial h}{\partial z} \cdot \frac{\partial h}{\partial \bar{z}},
$$

we get that

$$
\frac{\partial h}{\partial z} \cdot \frac{\partial h}{\partial \bar{z}}=0 \text { in } \Omega .
$$

If $\partial h / \partial z \neq 0$ on some open, nonempty subset $\Omega^{\prime}$ of $\Omega$, the function $\partial h / \partial \bar{z}$ is zero on $\Omega^{\prime}$ and harmonic in $\Omega$; then $\partial h / \partial \bar{z}=0$ in $\Omega$ (a fast proof of this is based on the following fact: if $u$ is a real harmonic function in a simply connected open subset $\Omega_{1}$ of the plane, and if $u$ is zero in a closed disk $K$ contained in $\Omega_{1}$, then, taking a conjugate harmonic function $v$ of $u$ defined on $\Omega_{1}$, the analytic function $\exp (u+i v)$ must be a constant in $\Omega_{1}$ by maximum principle applied to $D$ and analytic continuation to $\left.\Omega_{1}\right)$. Hence $\partial h / \partial z \not \equiv$ in $\boldsymbol{\Omega}$ implies that $h$ is analytic in $\Omega$. The same reasoning proves that if $\partial h / \partial \bar{z} \not z$ 0 , then $h$ is antianalytic in $\Omega$, and this ends the proof of the Lemma.

4. Proof of the Theorem. We prove first (i) $\Rightarrow$ (ii). If the law of the random variable $X$ is $\mu_{\omega}$, there exists $\tilde{f}(\omega)$ in $P$ such that $\mu_{\tilde{f}(\omega)}$ is the law of $f(X)$. The function $\tilde{f}: P \rightarrow P$ must satisfy:

$$
\int_{-\infty}^{+\infty} \exp [\operatorname{itf}(x)] \mu_{\omega}(d x)=\exp [i t \tilde{f}(\omega)]
$$

for all $t \geqslant 0$ and all $\omega$ in $P$.

In the integral of (5), we write

$$
x=i \frac{1+e^{i \theta}}{1-e^{i \theta}} .
$$

This function from $\mathbf{R}$ onto $\mathbf{T} \backslash\{0\}$ changes the measure $\mu_{\omega}$ in $P_{z}(\theta) d \theta / 2 \pi$ where $z=(i \omega+1) /(i \omega-1) \in U$. We define $g$ on $\mathbf{T} \backslash\{0\}$ and $\tilde{g}$ on $U$ by

$$
g\left(e^{i \theta}\right)=f\left(i \frac{1+e^{i \theta}}{1-e^{i \theta}}\right) \text { and } \tilde{g}=G \tilde{f} .
$$

The relation (5) becomes: 


$$
\frac{1}{2 \pi} \int_{-\pi}^{+\pi} \exp \left[i \operatorname{tg}\left(e^{i \theta}\right)\right] P_{z}(\theta) d \theta=\exp [i \operatorname{tg}(z)]
$$

for all $t>0$ and all $z$ in $U$.

(6) implies that the function $\exp [i t \tilde{g}(z)]$ is harmonic in $U$ for all $t>0$ (see [8, Theorem 11.8]), and by the Lemma, $\tilde{g}$ is either analytic or antianalytic in U.

Suppose first that $\tilde{g}$ is antianalytic. Then we take $\varepsilon=-1$ and have to prove that $-f$ has the indicated form by (ii) in the statement of the Theorem. Hermitian symmetry of Fourier transforms of real measures implies easily that $\widetilde{(-f)}=-\overline{\tilde{f}}$. Hence $G \widetilde{(-g)}$ is analytic. So, without loss of generality, we may suppose that $\tilde{\boldsymbol{g}}$ is analytic.

Since $\operatorname{Im} \tilde{g}>0$, the function $\exp [i t \tilde{g}(z)]$ belongs to the space $H^{\infty}$ of bounded holomorphic functions in $U$ for all $t>0$. This implies that

$$
\exp \left[i t \tilde{g}\left(r e^{i \theta}\right)\right] \underset{r \uparrow 1}{\rightarrow} \exp \left[i \operatorname{tg}\left(e^{i \theta}\right)\right]
$$

for fixed $t$ and for almost all $\theta$ of $\mathbf{T}$, by (6), using the corollary of the Theorem 17.12 of [8]. Taking $t$ as an integer $n>0$, (7) gives $\lim _{r \uparrow 1} \tilde{g}\left(r e^{i \theta}\right)-$ $g\left(e^{i \theta}\right) \equiv 0$ modulo $2 \pi n$, except on a subset $A_{n}$ of $\mathbf{T}$ with measure zero. So $\lim _{r \uparrow 1} \tilde{g}\left(r e^{i \theta}\right)-g\left(e^{i \theta}\right) \equiv 0$ modulo $2 \pi n$ for any positive integer $n$ and any $\theta$ outside of $A=\cup_{n=1}^{\infty} A_{n}$. Since the measure of $A$ is zero, we get

$$
\tilde{g}\left(r e^{i \theta}\right) \underset{r \uparrow 1}{\rightarrow} g\left(e^{i \theta}\right)
$$

for almost all $\boldsymbol{\theta}$ of $\mathbf{T}$.

Now $\operatorname{Im} \tilde{\mathbf{g}}(z)$ is a positive harmonic function in $U$. So, from Herglotz' Theorem (see [8, Theorem 11.19]) there exists a positive bounded measure $\nu$ on $\mathbf{T}$ such that

$$
\operatorname{Im} \tilde{g}(z)=\int_{-\pi}^{+\pi} P_{z}(\theta) \nu(d \theta)
$$

for all $z$ in $U$.

From Theorem 11.10 of [8], (9) gives

$$
\operatorname{Im} \tilde{g}\left(r e^{i \theta}\right) \underset{r \uparrow 1}{\rightarrow} \frac{d \nu}{d \theta}
$$

for almost all $\theta$ of $\mathbf{T}$, where $d \nu / d \theta$ is the Radon-Nikodým derivative of $\nu$ with respect to the Haar measure $d \theta$ of $\mathbf{T}$.

Analyticity of $\tilde{g}$ and equality (9) imply that there exists a real number $\alpha$ such that

$$
\tilde{g}(z)=\alpha+i \int_{-\pi}^{+\pi} \frac{e^{i \theta}+z}{e^{i \theta}-z} \nu(d \theta)
$$

for all $z$ in $U$.

Since $g\left(e^{i \theta}\right)$ is real, (8) and (10) imply that $d \nu / d \theta=0$ almost everywhere; that is to say, $\nu$ is singular with respect to the measure $d \theta$.

To come back to $f$ and $\tilde{f}$, we denote $k=\nu(\{0\})$ and consider the function from $\mathbf{T} \backslash\{0\}$ to $\mathbf{R}$ defined by 


$$
\gamma=i \frac{1+e^{i \theta}}{1-e^{i \theta}} .
$$

The measure $\nu$ restricted to $T \backslash\{0\}$ is carried by the function (12) to a positive bounded measure $\mu$ on $\mathbf{R}$ : thus it is trivial to check that $\mu$ is singular with respect to the Lebesgue measure on $\mathbf{R}$. (11) becomes:

$$
\tilde{f}(\omega)=F \tilde{g}(\omega)=k \omega+\alpha-\int_{-\infty}^{+\infty} \frac{\gamma \omega+1}{\omega-\gamma} \mu(d \gamma)
$$

for all $\omega$ in $P$.

Exploiting (8), we get the desired formula (ii). Note that the last part of the Theorem is a trivial consequence of (5) and (13).

We prove now that (ii) $\Rightarrow$ (i). Without loss of generality we suppose that $\varepsilon=+1$. Denote by $\nu$ the measure on $\mathbf{T}$ whose restriction to $\mathbf{T} \backslash\{0\}$ is carried from $\mu$ by the inverse function of (12), and such that $\nu(\{0\})=k$. Consider $\tilde{g}$ defined by (11) in $U$. Clearly $\operatorname{Im} \tilde{g}(z)>0$ in $U$ and for fixed positive $t$, $\exp [i t \tilde{g}]$ belongs to $H^{\infty}$ and is the Poisson integral of some function $\phi_{t}\left(e^{i \theta}\right)$ of $L^{\infty}(T)$ (see Theorem 17.12 of [8] and its corollary). Furthermore

$$
\exp \left[i t \tilde{g}\left(r e^{i \theta}\right)\right] \underset{r \uparrow 1}{\rightarrow} \phi_{t}\left(e^{i \theta}\right)
$$

for almost all $\theta$ of $\mathbf{T}$.

The definition of $f$ implies that

$$
\phi_{t}\left(e^{i \theta}\right)=\exp \left[\operatorname{itg}\left(e^{i \theta}\right)\right]
$$

for almost all $\theta$ of $\mathbf{T}$, where

$$
g\left(e^{i \theta}\right)=f\left(i \frac{1+e^{i \theta}}{1-e^{i \theta}}\right) .
$$

Then (6) is true, and thus it is easy to come back to (5), which gives (i).

6. Proof of the Corollary. It is enough to prove that if $\mu$ is singular, positive and bounded,

$$
f(x)=x-\lim _{y \downarrow 0} \int_{-\infty}^{+\infty} \frac{(x+i y) \gamma+1}{x+i y-\gamma} \mu(d \gamma)
$$

preserves Lebesgue measure.

Let $a<b$ and denote $g(x)=\mathbf{1}_{[a, b]}(x)$. We have to prove

$$
\int_{-\infty}^{+\infty} g[f(x)] d x=b-a .
$$

From the Theorem, we know that

$$
\int_{-\infty}^{+\infty} g[f(x)] \mu_{\omega}(d x)=\int_{a}^{b} \mu_{\tilde{f}(\omega)}(d x)
$$

for any $\omega$ in $P$. We take $\omega=i k$, with $k>0$. Note that 


$$
\begin{aligned}
& \operatorname{Im} \tilde{f}(i k)=k+k \int_{-\infty}^{+\infty} \frac{\gamma^{2}+1}{\gamma^{2}+k^{2}} \mu(d \gamma) \\
& \operatorname{Re} \tilde{f}(i k)=\int_{-\infty}^{+\infty} \frac{\gamma\left(1-k^{2}\right)}{\gamma^{2}+k^{2}} \mu(d \gamma) .
\end{aligned}
$$

We get

$$
\lim _{k \rightarrow \infty} \frac{1}{k} \operatorname{Im} \tilde{f}(i k)=1 \text { and } \lim _{k \rightarrow \infty} \operatorname{Re} \tilde{f}(i k)=-\mu(\mathbf{R})
$$

by dominated convergence. A routine calculation gives:

$$
\int_{a}^{b} \mu_{\tilde{f}(\omega)}(d x)=\frac{1}{\pi} \operatorname{Arctg} \frac{b+\operatorname{Re} \tilde{f}(i k)}{\operatorname{Im} \tilde{f}(i k)}-\frac{1}{\pi} \operatorname{Arctg} \frac{a+\operatorname{Re} \tilde{f}(i k)}{\operatorname{Im} \tilde{f}(i k)}
$$

so

$$
\lim _{k \rightarrow \infty} k \int_{a}^{b} \mu_{\tilde{f}(\omega)}(d x)=\frac{1}{\pi}(b-a) .
$$

Considering now the first member of (15):

$$
\lim _{k \rightarrow \infty} k \int_{-\infty}^{+\infty} g[f(x)] \frac{k}{\pi\left(x^{2}+k^{2}\right)} d x=\int_{-\infty}^{+\infty} g[f(x)] d x .
$$

(15), (16) and (17) give (14), and the Corollary is proved.

7. Remarks. (1) A "brownian motion" interpretation of the Theorem goes as follows. Let $b(t)$ a standard brownian motion in the complex plane such that $b(0)=\omega$ in $P$. Denote $T=\inf \{t ; b(t)$ belongs to real axis $\}$. As is well known, the distribution of $\operatorname{Re} b(T)$ is $\mu_{\omega}$ (see [3, p. 172] for a proof). If $\tilde{f}$ is as in the Theorem, with $\varepsilon=1$, the new process $\tilde{f}(b(t))$, restricted to $[0, T]$ has the same property.

One can use this idea and a remark made by Harry Kesten (quoted in [4]) to give a second proof of the second part (ii) $\Rightarrow$ (i) of the Theorem. Denote by $h(t)$ the (random) inverse of

$$
\int_{0}^{t}\left|f^{\prime}(b(s))\right|^{2} d s
$$

defined in $\left[0, h^{-1}(T)\right]$. Then a Paul Lévy Theorem implies that $t \mapsto f[b(h(t))]$ $=b_{1}(t)$ is a brownian motion in $\left[0, h^{-1}(T)\right]$. But $f$ is never real in $P$, since

$$
\operatorname{Im} f(x+i y)=k y+y \int_{-\infty}^{+\infty} \frac{\gamma^{2}+1}{(x-\gamma)^{2}+y^{2}} \mu(d \gamma)
$$

Hence $f(b(T))$ is the point of $\mathbf{R}$ where $b_{1}(t)$ strikes $\mathbf{R}$ for the first time, with the distribution $\mu_{f(\omega)}$.

(2) If $f$ and $\tilde{f}$ are as in the Corollary, with $\varepsilon=1$, one can check that there exists a probability distribution $p$ on $\mathbf{R}$ such that

$$
\frac{1}{\tilde{f}(\omega)}=\int_{-\infty}^{+\infty} \frac{p(d \gamma)}{\omega-\gamma}
$$


The proof of this can be easily gotten from Lemma 2.2, p. 24 of [9]. Since iterates of $\tilde{f}$ have the same property, we hope to explain and generalise some day the results of [1] in terms of a Markov chain defined on $\mathbf{R}$ properly associated to the probability $p$.

(3) If $f$ and $\tilde{f}$ are as in the Theorem, with $\varepsilon=+1$, the transform by $f$ of a bounded complex measure on $\mathbf{R}$ with a rational density has also a rational density. Such a density can be easily computed with the following remark: if $n$ is a nonnegative integer and $\omega$ is in $P$, the transform by $f$ of the measure $(x-\omega)^{-n-1} d x$ is

$$
\frac{1}{n !}\left(\frac{\partial}{\partial \omega}\right)^{n}(x-\tilde{f}(\omega))^{-1} d x .
$$

A proof can be given by residues computation of:

$$
\int_{-\infty}^{+\infty} \exp [\operatorname{itf}(x)](x-\omega)^{-n-1} d x
$$

This method gives a third proof of the second part (ii) $\Rightarrow$ (i) of the Theorem. A similar remark can be done if $k>0$ and if the measure on $\mathbf{R}$ to be transformed by $f$ is an unbounded Radon measure with rational density. But the computation of the density of the transformed measure is more complicated.

(4) Let $X$ be a random variable on $\mathbf{R}$ and $\mu_{a, b}$ the law of $a X+b$. Denote $\mu=\mu_{0,1}$ and $\mathscr{F}(\mu)$ the set of measurable functions $f$ from $\mathbf{R}$ to $\mathbf{R}$ such that for any $a \neq 0$ and $b$ real, the law of $f(a X+b)$ belongs to $\left\{\mu_{\alpha, \beta} ; \alpha \neq 0, \beta \in \mathbf{R}\right\}$. We have characterised

$$
\mathscr{F}\left(\frac{1}{\pi} \frac{d x}{1+x^{2}}\right)
$$

It is easy to check that

$$
\mathscr{F}\left(\mathbf{1}_{[0,1]}(x) d x\right)=\{a x+b ; a \neq 0, b \in \mathbf{R}\} .
$$

This is also true for $\mathscr{F}\left(e^{-x^{2} / 2} d x / \sqrt{2 \pi}\right)$; a generalisation of this fact will appear in Comptes Rendus de l'Académie des Sciences. The proof is easy: we apply heat operator (instead of Laplacian) to the equation which replaces (5) for Gaussian measure.

\section{REFERENCES}

1. R. L. Adler and B. Weiss, The ergodic infinite measure preserving transformation of Boole, Israel J. Math. 16 (1973), 263-278.

2. P. L. Duren, Theory of $H^{p}$ spaces, Academic Press, New York and London, 1970.

3. W. Feller, An introduction to probability theory and its applications. II, 1st ed., Wiley, New York, 1966.

4. J. H. B. Kempermann, The ergodic behavior of a class of real transformations, Stochastic Processes and Related Topics. I (Proc. Summer Research Inst. on Statistical Inference for Stochastic Processes, Indiana University, Bloomington, 1974), Academic Press, New York, 1975, pp. 249-258.

5. F. B. Knight, A characterisation of Cauchy type, Proc. Amer. Math. Soc. 55 (1976), 130-135.

6. F. B. Knight and P. A. Meyer, Une caractérisation de la loi de Cauchy, Z. Wahrscheinlichkeitstheorie und Verw. Gebiete 34 (1976), 129-134. 
7. G. Pölya and G. Szegö, Problems and theorems in analysis. I, II, Problem 118.1, SpringerVerlag, Betlin and New York, 1972.

8. W. Rudin, Real and complex analysis, McGraw-Hill, New York, 1966.

9. J. A. Shohat and J. D. Tamarkin, The problem of moments, Math. Surveys, no. 1, Amer. Math. Soc., Providence, R. I., 1963.

10. E. J. Williams, Cauchy-distributed functions and a characterisation of the Cauchy distribution, Ann. of Math. Stat. 4 (1969), 1083-1085.

Dípartendant dB Mathíachouges, Universtré PaUl-Sabatier, 118, Route de Nareonne, 31077 Toulouse, France 